\title{
Synchronous Fluorometric Determination of Anthracene in Polynuclear Aromatic Chemicals after Zone-melting Separation Using Dibenzofuran as a Medium
}

\author{
Masaki Tachibana and Motohisa Furusawa \\ Faculty of Engineering, Yamanashi University, Kofu 400
}

\begin{abstract}
The applicability of the zone-melting technique using dibenzofuran as a medium to the separation of small amounts of anthracene in polynuclear aromatic chemicals has been studied. After $100 \mathrm{mg}$ (or $20 \mathrm{mg}$ ) of the chemicals containing anthracene had been mixed with $4 \mathrm{~g}$ of purified dibenzofuran, 20 zone-passes were performed. Most of the compounds and the concomitant impurities could be eliminated from the beginning part of the zonemolten ingot, in which anthracene is concentrated. By this technique, anthracene was separated from chemicals such as pyrene, benz [a] anthracene, 9-fluorenone, and 9,10-phenanthrenequinone up to $100 \mathrm{mg}$, and perylene and anthraquinone up to $20 \mathrm{mg}$. The zone-molten ingot was cut into halves and the beginning half was dissolved in xylene. Anthracene separated in this half could be determined by synchronous fluorescence spectrometry without removal of dibenzofuran. The recovery of anthracene was $93-95 \%$. The determination limit in the first four chemicals listed was $2 \mathrm{ppm}$ and in the latter two the limit was $10 \mathrm{ppm}$.
\end{abstract}

Keywords Anthracene, zone-melting separation, dibenzofuran, polynuclear aromatic chemicals, synchronous fluorescence spectrometry

Since anthracene is one of the main components in coal tar, many polynuclear aromatic chemicals derived from tar often contain anthracene as an impurity. Therefore, it is important to determine small amounts of anthracene in such chemicals as pyrene, benz[a]anthracene, anthraquinone, 9-fluorenone, etc. Though several determination methods of anthracene in anthraquinone $e^{1-6}$ and pyrene ${ }^{7}$ have been developed, few methods for the other compounds have been presented. This is largely attributable to the difficulty in separating small amounts of anthracene from the matrix compounds and the concomitant impurities, most of which have large effects on the spectral measurements of anthracene.

The authors have recently reported that trace amounts of anthracene in fluoranthene can be determined after separation by use of a zone-melting technique using dibenzofuran as a medium. ${ }^{8}$ In the present paper, applicability of this technique has been further studied for the separation of anthracene in other chemicals. Small amounts of anthracene could be separated from pyrene, benz [a] anthracene, 9-fluorenone, 9,10-phenanthrenequinone, perylene, and anthraquinone and its interfering impurities by the zonemelting technique followed by synchronous fluorometric determination. Anthracene, however, was inseparable from naphthacene, carbazole, and $5 \mathrm{H}$ benzo $[b]$ carbazole because the distribution coefficients of these three compounds in dibenzofuran are close to or greater than unity. Anthracene was separated from chrysene, but could not be determined because chrysene contains $5 \boldsymbol{H}$-benzo $[b]$ carbazole as an impurity.

\section{Experimental}

\section{Reagents}

Commercially available dibenzofuran was purified by combining several methods before use as a zonemelting medium. The process of purification has been described in detail in a previous paper. ${ }^{8}$ Pyrene and anthraquinone were zone-refined after removal of anthracene by Diels-Alder reaction with maleic anhydride. Chrysene was dissolved in benzene and shaken with $87 \%$ sulfuric acid to remove $5 \mathrm{H}$ benzo $[b]$ carbazole. The chrysene obtained after evaporation of the benzene was further purified in a manner similar to that used for pyrene. Benz $[a]-$ anthracene, 9-fluorenone, and 9,10-phenanthrenequinone were purified only by zone melting. Xylene was of guaranteed reagent grade and used without further purification.

\section{Apparatus}

Zone meltings were carried out on a Shibayama SS950 high-speed zone refiner, which has been already 
described in detail elsewhere. 9 Synchronous fluorescence spectra were measured with a Hitachi 650-40 fluorescence spectrophotometer equipped with a Hitachi 056 recorder. The spectral bandpass was always set at $2 \mathrm{~nm}$. A 150-W xenon lamp was used as an exciting source. A $10 \times 10 \times 45 \mathrm{~mm}^{3}$ quartz cell was used for the spectral measurements of solutions.

\section{Procedure}

A mixture of $100 \mathrm{mg}$ (or $20 \mathrm{mg}$ ) of a sample and $4 \mathrm{~g}$ of the purified dibenzofuran was charged in a glass tube with an i.d. of $4 \mathrm{~mm}$. The zone-pass was then repeated 20 times. The six molten zones, each about 30 $\mathrm{mm}$ long, were set to travel upward at a speed of 100 $\mathrm{mm} / \mathrm{h}$. During the travel, the zones were stirred by spinning the tube at $20 \mathrm{~s}^{-1}$, with reversal of the rotation direction at intervals of $1.0 \mathrm{~s}$. The zone-molten ingot was cut into halves exactly, and then the lower half was dissolved in $20 \mathrm{ml}$ of xylene. The synchronous fluorescence spectrum of the solution was measured with a wavelength interval of $6 \mathrm{~nm}$. Anthracene was determined from the intensity at $384 \mathrm{~nm}$ obtained by the base line method. The synchronous fluorescence intensity of an anthracene solution $(0.100 \mu \mathrm{g} / \mathrm{ml})$ was used as the standard. The calibration curve was prepared by using anthracene solutions $(0-0.1 \mu \mathrm{g} / \mathrm{ml})$ containing dibenzofuran. The incomplete recovery of anthracene was corrected for by using the reciprocal of 0.95 (or 0.93 in the case of $20 \mathrm{mg}$ ) as the correction factor.

\section{Results and Discussion}

\section{Separation of anthracene from polynuclear aromatic compounds}

Each of the compounds of interest to separate was mixed with dibenzofuran in the concentration range of about $0.1-3 \%$. The mixture was charged in a glass tube, and zone melting was carried out with an upward travel under constant stirring. After the 20 zonepasses, the zone-molten ingot was divided into 8-15 portions and the quantity of the compound in the portions was measured by ultraviolet spectrophotometry. From the results of these experiments, concentration profiles for the compounds in dibenzofuran were prepared. The resulting profiles could be roughly classified into three groups. The first group consists of the profiles for pyrene, benz $[a]$ anthracene, 9,10phenanthrenequinone, and 9-fluorenone, which are shown in Fig. 1. These compounds can be fully accumulated on the end part of the zone-molten ingot up to about $3 \%$, though the effectiveness of the accumulation gradually decreases with an increase in their content. The concentration profiles for anthraquinone, perylene, and chrysene, which belong to the second group, are shown in Fig. 2. The profiles are similar to those for the first group in the concentration range below $0.5 \%$. In higher concentration, however,

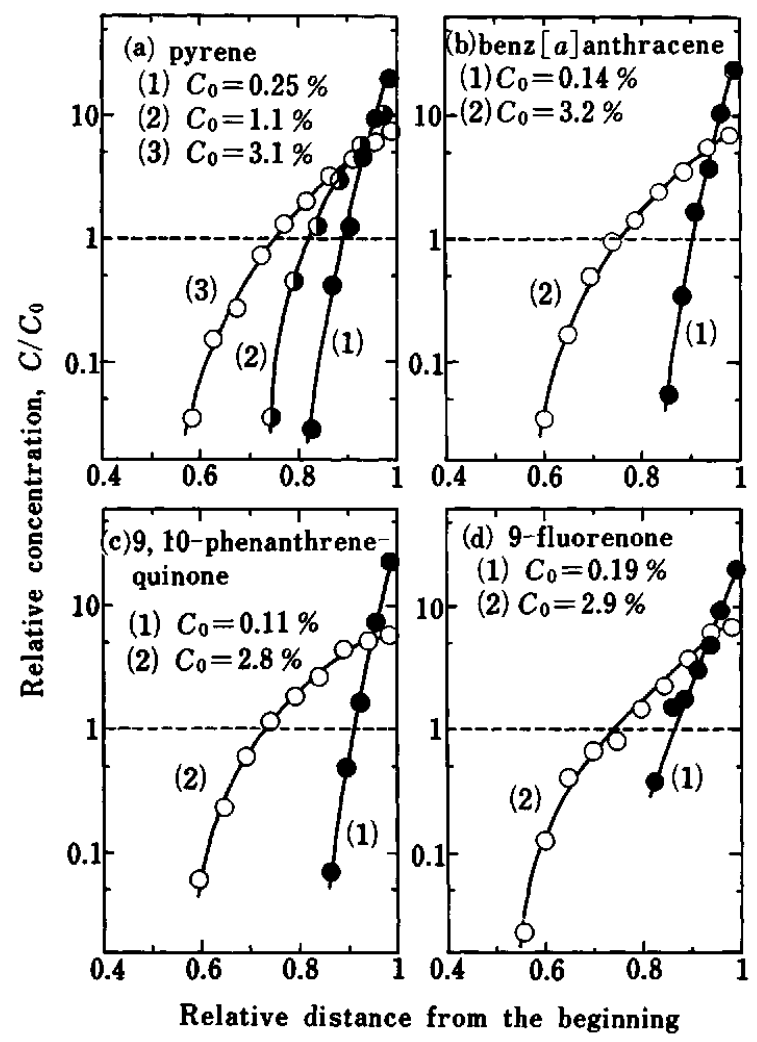

Fig. 1 Concentration profiles for pyrene, benz $[a]$ anthracene, 9,10-phenanthrenequinone, and 9-fluorenone in dibenzofuran. $C_{0}$ and $C$ are initial and final concentrations, respectively.

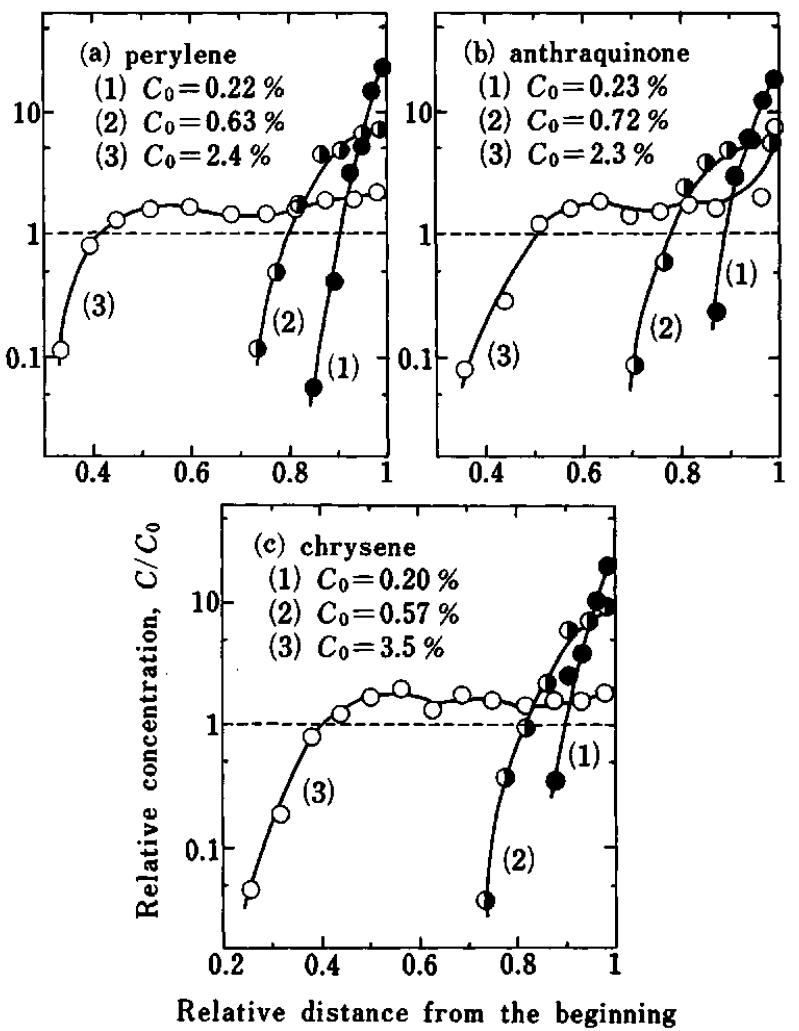

Fig. 2 Concentration profiles for perylene, anthraquinone, and chrysene in dibenzofuran. 


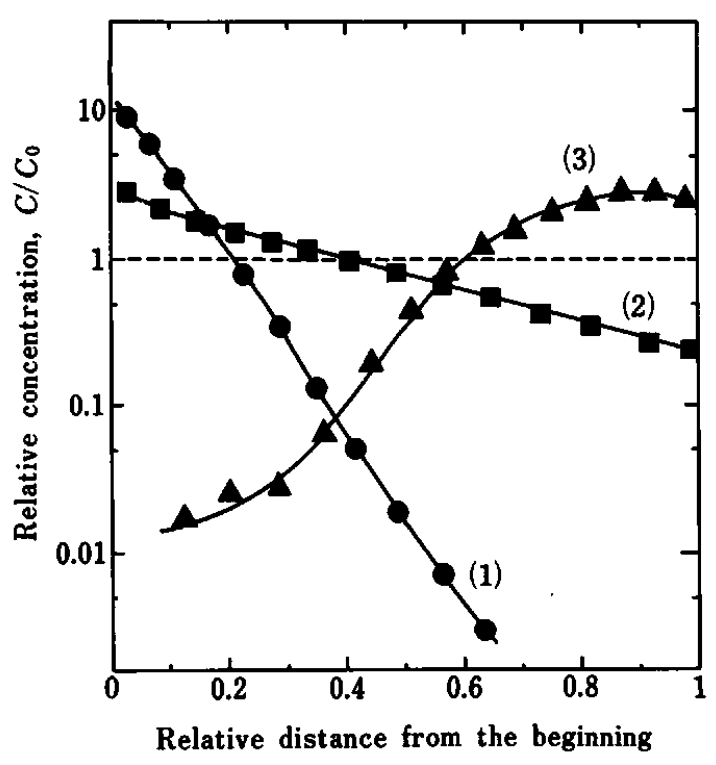

Fig. 3 Concentration profiles for carbazole, $5 H$-benzo $[b]$ carbazole, and naphthacene in dibenzofuran. (1) Carbazole, $C_{0}=0.10 \%$; (2) $5 H$-benzo $[b]$ carbazole, $C_{0}=0.11 \%$; (3) naphthacene, $C_{0}=0.09 \%$.

these three compounds cannot be effectively accumulated on the end part. The profiles for naphthacene, carbazole, and $5 H$-benzo $[b]$ carbazole, which are classified into the third group, are shown in Fig. 3. Naphthacene cannot be completely accumulated on the end part, even in low concentration. Carbazole and $5 H$-benzo $[b]$ carbazole are concentrated toward the beginning part of the ingot, similarly to anthracene. The results of these experiments indicate that the compounds which belong to the first and second groups can be effectively removed up to $100 \mathrm{mg}$ and $20 \mathrm{mg}$ respectively, while the third group cannot be removed.

\section{Recovery of anthracene}

When zone melting of dibenzofuran containing anthracene alone has been carried out under the same conditions as above, the recovery of anthracene has been observed to be approximately $92 \%$ in the lower half of the zone-molten ingot. ${ }^{10}$ In the presence of fluoranthene, however, the recovery has slightly increased with an increase in the content of fluoranthene. ${ }^{8}$ Therefore, such effects were also studied in the presence of each compound listed in Figs. 1 and 2. Table 1 shows the recovery of anthracene measured in the presence of $100 \mathrm{mg}$ or $20 \mathrm{mg}$ of the compounds. Under the conditions of these experiments, the recovery was dependent on the quantities of the compounds, but practically independent of the species. The recovery is approximately $95 \%$ when $100 \mathrm{mg}$ of the compounds are incorporated, and $93 \%$ when $20 \mathrm{mg}$ are. The incomplete recovery, however, can be corrected for by use of the reciprocal of 0.95 or 0.93 as the correction factor.
Table 1 Recovery of anthracene in the presence of large amounts of polynuclear aromatic compounds

\begin{tabular}{|c|c|c|c|c|}
\hline $\begin{array}{l}\text { Anthracene } \\
\text { added/ug }\end{array}$ & $\begin{array}{l}\text { Compound } \\
\text { added/mg }\end{array}$ & & $\begin{array}{c}\text { Anthracene } \\
\text { found/ } \mu g\end{array}$ & $\begin{array}{c}\text { Recovery, } \\
\%\end{array}$ \\
\hline 2.14 & 9-fluorenone & 101 & $2.0_{2}$ & 94.4 \\
\hline 2.28 & 9,10 -phen- & 100 & 2.20 & 96.5 \\
\hline 2.14 & $\begin{array}{l}\text { anthrene- } \\
\text { quinone }\end{array}$ & 101 & $2.0_{1}$ & $93 .$, \\
\hline 2.28 & $\begin{array}{l}\text { benz }[a]- \\
\text { anthracene }\end{array}$ & 102 & 2.1, & 96.1 \\
\hline 2.13 & pyrene & 101 & 2.04 & 95.8 \\
\hline 2.28 & $"$ & 101 & $2.1_{8}$ & $\begin{array}{r}95.6 \\
\bar{x}=95.4\end{array}$ \\
\hline 2.14 & " & 21 & $2.0_{0}$ & 93.5 \\
\hline 2.28 & " & 21 & $2.1_{2}$ & 93.0 \\
\hline 2.14 & chrysene & 21 & 2.00 & 93.s \\
\hline 2.13 & anthraquinone & e 21 & $2.0_{0}$ & 93.9 \\
\hline 2.28 & " & 21 & $2.1_{2}$ & $\begin{array}{r}93.0 \\
\bar{x}=93.4\end{array}$ \\
\hline 2.14 & none & & 1.98 & 92.5 \\
\hline
\end{tabular}

\section{Calibration curve}

Though dibenzofuran has a little effect on synchronous fluorescence measurements of anthracene, the effect can be corrected for by use of a calibration curve prepared in the presence of dibenzofuran. ${ }^{8}$ Synchronous fluorescence spectra of xylene solutions of anthracene $(0-0.1 \mu \mathrm{g} / \mathrm{ml})$ containing dibenzofuran $(100 \mathrm{mg} / \mathrm{ml})$ were measured with a wavelength interval of $6 \mathrm{~nm}$. The intensities at $384 \mathrm{~nm}$ obtained by the base line method were employed to prepare the calibration curve for anthracene. The synchronous fluorescence intensity of a $0.100 \mu \mathrm{g} / \mathrm{ml}$ anthracene solution in xylene was used as the standard. The curve thus obtained was linear over the above concentration range.

\section{Effect of diverse substances}

Although no separation of anthracene from carbazole has been achieved by the zone-melting technique, the effect of carbazole on the spectral measurement of anthracene is relatively small: negligible up to approximately 500-fold amount. ${ }^{11}$ Approximately 20fold amount of naphthacene has no effect on the determination of anthracene. On the other hand, $5 \mathrm{H}$ benzo $[b]$ carbazole greatly affects the determination of anthracene, because the two synchronous fluorescence spectra overlap each other. Even at the concentration of $0.01 \mu \mathrm{g} / \mathrm{ml}$, the presence of $5 H$-benzo $[b]$ carbazole interfered with the measurement of synchronous fluorescence intensity of a $0.1 \mu \mathrm{g} / \mathrm{ml}$ anthracene solution. Therefore, this method is not applicable to such chemicals as chrysene, which contains $5 \mathrm{H}$ benzo $[b]$ carbazole as an impurity. Each of the interfering compounds listed in Figs. 1 and 2 is easily removable from anthracene by the zone-melting technique. Nevertheless, the effectiveness of the 


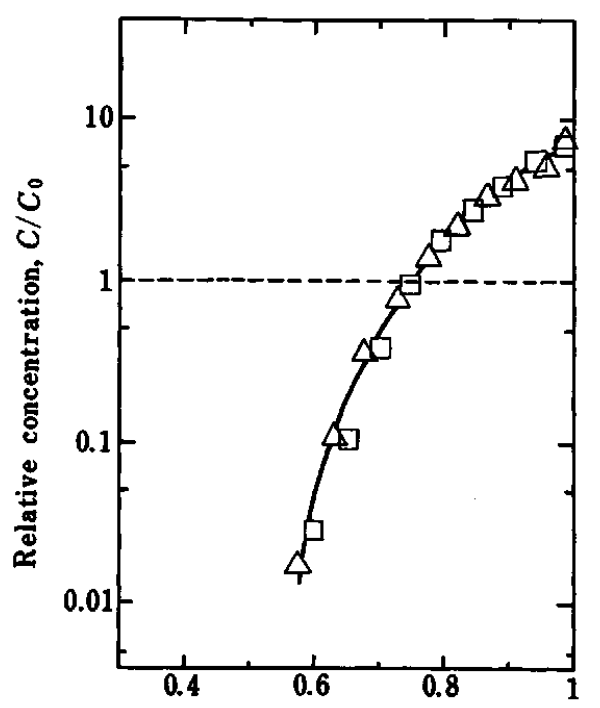

Relative distance from the beginning

Fig. 4 Concentration profile for perylene in dibenzofuran in the presence of large amounts of pyrene or fluoranthene. $C_{0}=0.16 \% ; \square$ pyrene $(104 \mathrm{mg} / 4 \mathrm{~g}) ; \Delta$ fluoranthene (106 $\mathrm{mg} / 4 \mathrm{~g})$.

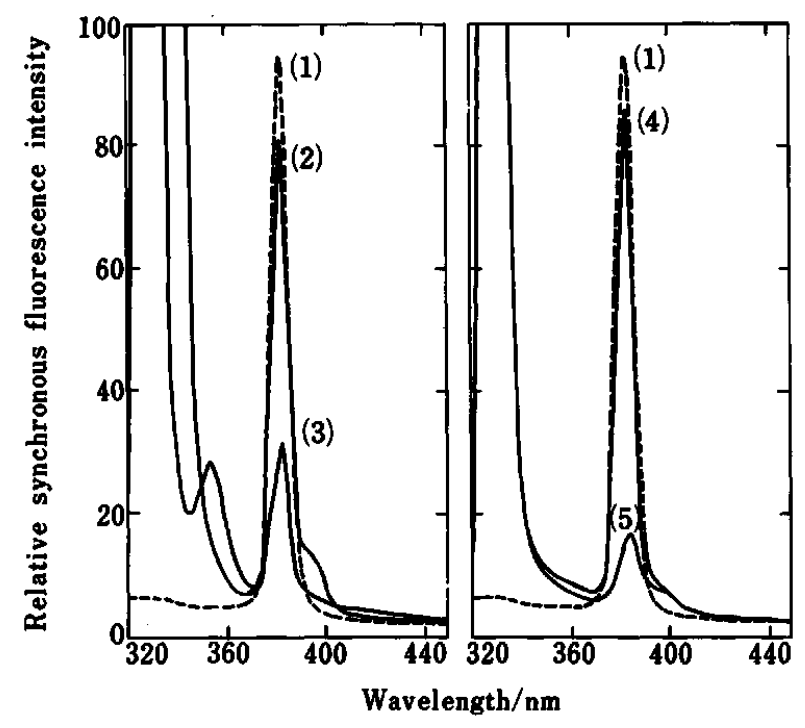

Fig. 5 Synchronous fluorescence spectra of the solutions used for the determination in each practical sample of pyrene and anthraquinone. Wavelength interval $(\Delta \lambda), 6$ $\mathrm{nm}$; solvent, xylene; (1) anthracene solution $(0.100 \mu \mathrm{g} / \mathrm{ml})$; (2) and (3), used for the determination in pyrene; (2) diluted 5 times; (4) and (5), used for the determination in anthraquinone.

removal decreased slightly in the coexistence of large amounts of the other substances. As an example, the concentration profile for perylene in the presence of approximately $100 \mathrm{mg}$ of fluoranthene and pyrene is illustrated in Fig. 4. Owing to the interference of fluoranthene or pyrene, the effectiveness of the removal
Table 2 Analytical results for anthracene in synthetic mixtures of pyrene and anthraquinone

\begin{tabular}{rccc}
\hline No. & $\begin{array}{c}\text { Content, } \\
\text { ppm }\end{array}$ & $\begin{array}{c}\text { Relative synchronous } \\
\text { fluorescence intensity }\end{array}$ & $\begin{array}{c}\text { Found, } \\
\text { ppm }\end{array}$ \\
\hline 1 & 2.2 & 9.1 & 2.1 \\
2 & 6.0 & 25.2 & 5.8 \\
3 & 11.2 & 47.0 & 10.9 \\
4 & 15.0 & 62.4 & 14.5 \\
5 & 22.4 & 93.9 & 21.7 \\
6 & 10.0 & 10.8 & 11.4 \\
7 & 26.8 & 25.4 & 26.7 \\
8 & 49.9 & 46.2 & 48.7 \\
9 & 66.5 & 63.0 & 66.3 \\
10 & 99.7 & 96.2 & 101 \\
\hline
\end{tabular}

Numbers 1-5, mixtures of pyrene; Nos. 6-10, anthraquinone.

a. Synchronous fluorescence intensity of an anthracene solution $(0.100 \mu \mathrm{g} / \mathrm{ml})$ was taken as 90 div.

decreases, compared with that of perylene alone as shown in Fig. 2 (a) (1). The compounds listed in Figs. 1 and 2 have no influence on the determination of anthracene in the presence of at least $2 \%$ in the chemicals as impurities.

\section{Determination of trace amounts of anthracene in pyrene}

Synthetic mixtures of pyrene containing anthracene and the other polynuclear aromatic compounds were prepared. Each mixture contains $0.6 \%$ of phenanthrene and fluoranthene, $0.01 \%$ of carbazole, chrysene, benz $[a]$ anthracene, naphthacene, perylene, anthraquinone, and 9,10-phenanthrenequinone. The analytical results for anthracene in the mixtures are shown in Table 2, Nos. 1-5. These results agree well with the individual content of anthracene in the mixtures. In addition, anthracene in two different pyrene samples obtained commercially was determined by the present method. The analytical results were $5 \mathrm{ppm}$ and 77 $\mathrm{ppm}$. The synchronous fluorescence spectra of the solutions used for the determination are illustrated in Figs. 5 (2) and (3). The shapes of the two spectra agree well with that of anthracene solution containing dibenzofuran, which is also shown in Fig. 5 (1). In these solutions, no absorption based on interfering impurities was observed at the characteristic wavelength of anthracene. Therefore, trace amounts of anthracene in pyrene can be determined by this method.

\section{Determination of small amounts of anthracene in anthraquinone}

The analytical results in synthetic mixtures of anthraquinone are shown in Table 2, Nos. 6-10. Each mixture contains $2.0 \%$ of 9,10 -phenanthrenequinone, $0.6 \%$ of phenanthrene, carbazole, 9-fluorenone, and acenaphthenequinone, and $0.1 \%$ of pyrene, fluoranthene, acenaphthene, and fluorene. In addition, the amounts of anthracene in two different commercial 


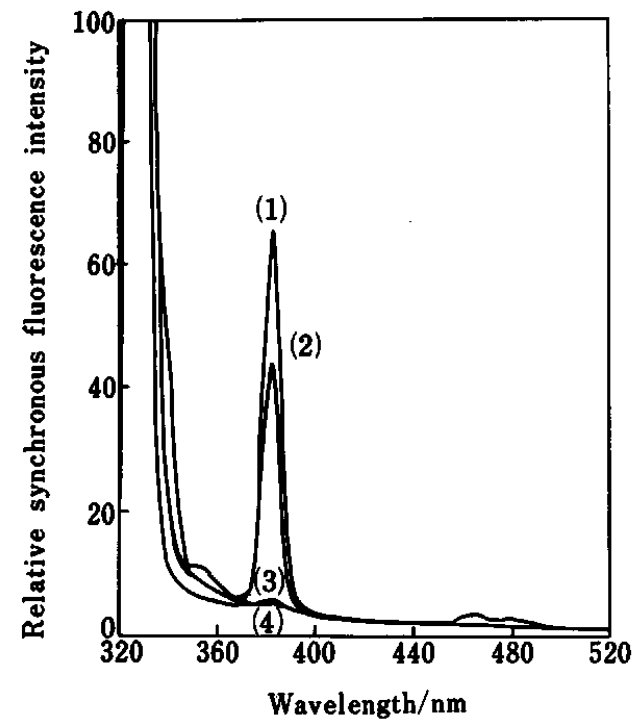

Fig. 6 The spectra of the solutions used for the determination in each practical sample of benz $[a]$ anthracene, perylene, 9-fluorenone, and 9,10-phenanthrenequinone. Solvent, xylene; $\Delta \lambda, 6 \mathrm{~nm}$; standard, intensity of an anthracene solution $(0.100 \mu \mathrm{g} / \mathrm{ml})$ was taken as $90 \mathrm{div}$.; (1) used for the determination in 9-fluorenone, diluted twice; (2) in benz [a] anthracene, diluted 5 times; (3) in 9,10-phenanthrenequinone; (4) in perylene.

anthraquinone were determined to be $13 \mathrm{ppm}$ and 99 ppm. The spectra used for the determination are shown in Figs. 5 (4) and (5), which are identical with that of anthracene shown in Fig. 5 (1) and are not affected by the absorption of the other impurities. These results for the synthetic mixtures and practical samples indicate that the present method is also applicable to the determination of small amounts of anthracene in anthraquinone.

\section{Determination in other polynuclear aromatic chemicals}

Anthracene in four commercially available chemicals, namely, benz [a] anthracene, 9-fluorenone, per- ylene, and 9,10-phenanthrenequinone was evaluated by the present method. The analytical results of anthracene in the benz $[a]$ anthracene and the 9fluorenone were $44 \mathrm{ppm}$ and $27 \mathrm{ppm}$, respectively. In the perylene and the 9,10-phenanthrenequinone, no anthracene could be detected by this method because the quantity in them was too small. The contents of anthracene in these two chemicals were less than 1 ppm. Figure 6 shows the synchronous fluorescence spectra of the solutions used for the determination in the four chemicals. No absorption based on the other impurities was observed in these solutions. From the results of these experiments, this method can be applied to the determination of anthracene in these polynuclear aromatic chemicals.

\section{References}

1. M. Furusawa, T. Takeuchi, K. Ogura and T. Hosoda, Kogyo Kagaku Zasshi, 68, 1203 (1965).

2. L. Ya. Kheifets, Zavod. Lab., 34, 801 (1968).

3. G. Grzegorzek-Halarewicz, Polish P., 57, 329 (1969).

4. M. Furusawa, T. Takeuchi, H. Fujiwara and T. Suzuki, Nippon Kagaku Kaishi, 1972, 1536.

5. L. A. Kogan, N. L. Sorokina and V. G. Nazarov, Zavod. Lab., 39, 675 (1973).

6. M. Furusawa, S. Iwasaki and Y. Matsuura, Nippon Kagaku Kaishi, 1974, 2228.

7. C. A. Parker, C. G. Hatchard and T. A. Joyce, Analyst [London] 90, 1 (1965).

8. M. Tachibana and M. Furusawa, Anal. Sci., 1, 77 (1985).

9. M. Furusawa and M. Tachibana, Bull. Chem. Soc. Jpn., 54, 2968 (1981).

10. M. Tachibana and M. Furusawa, Bull. Chem. Soc. Jpn, 56, 2254 (1983).

11. M. Furusawa, M. Tachibana and Y. Hayashi, Bunseki Kagaku, 31, 229 (1982).

(Received June 3, 1987)

(Accepted July 21, 1987) 